\title{
Molecular Docking and Dynamic Simulation of UDP-N-Acetylenolpyruvoylglucosamine Reductase (MurB) obtained from Mycobacterium tuberculosis using in silico Approach
}

\section{Mustafa Alhaji Isa and Mohammed Mustapha Mohammed}

Department of Microbiology, Faculty of Sciences, University of Maiduguri, Nigeria Corresponding author: mustafaisa@unimaid.edu.ng

\section{Abstract}

The UDP-N-acetylenolpyruvoylglucosamine reductase (MurB) catalyze the final steps of the UDP-N-acetylmuramic acid (UDPMurNAc) formation in the peptidoglycan biosynthesis pathway. The absence of this pathway in mammal made it an attractive target for drug development in Mycobacterium tuberculosis (MTB). In this study, the crystal structure of MurB from MTB (PDB Code: 5JZX and resolution of $2.2 \AA$ ) bound to FAD and $\mathrm{K}^{+}$was obtained from Protein Data Bank (PDB). A total of 2157 compounds with best binding conformations obtained from zinc database through virtual screening. These compounds further screened for drug-likeness, pharmacokinetic properties, physicochemical properties (Lipinski rule of five), and molecular docking analysis to obtained compounds with desirable therapeutic properties and good binding energies against MurB. Seven compounds (7) with minimum binding energies ranged between -11.80 and $-10.39 \mathrm{kcal} / \mathrm{mol}$ were selected, lower than the binding energy of FAD $(-10.06 \mathrm{kcal} / \mathrm{mol})$. Four compounds with best binding energies $($ ZINC19837204 $=-11.80 \mathrm{kcal} / \mathrm{mol}, \quad$ ZINC11839554 $=-11.47 \mathrm{kcal} / \mathrm{mol}$, ZINC14976552 $=-10.77 \mathrm{kcal} / \mathrm{mol}$ ) and ability to interact with the residues (ZINC12242812 $=-10.39 \mathrm{kcal} / \mathrm{mol}$ ) of the substrate binding site further selected for the molecular dynamic (MD) simulation analysis. The result of the MD simulation showed that all the four ligands formed stable complexes in the binding site of the MurB, during the 50ns MD simulation, when compared with the cofactor (FAD). Therefore, these compounds were proposed to be novel inhibitors of MTB after in vivo and in vitro validation.

Key Words: MurB, Ligand, Docking, MD simulation and Pharmacokinetic properties

\section{Introduction}

Tuberculosis (TB) is an infectious disease responsible for mortality and morbidity in this twenty-first century. It is a global public health threat that is considered the second highest cause of death, only next to human immunodeficiency virus (HIV) (WHO, 2016). Despite the presence of tuberculosis control programme, the disease poses a serious threat, due to the existence of multi-drug resistant tuberculosis (MDR-TB), extensively drug-resistant (XDR) 
and total drug-resistant (TDR). The rate of mortality as a result of tuberculosis is on the increase due to the emergence of HIV-TB co-infection (Jothieswari and Bhaskar-Reddy, 2015). The disease is transmitted widely within communities or societies through sneezing, coughing or staying with individuals having an active form of the infection. The disease is caused by MTB, with the lung as a primary target site for the organism, but subsequently may spread to the remaining organs of the body, such as bone, central nervous system, lymph node and genitourinary tract. Despite significant efforts made in controlling TB, the disease remains a substantial cause of mortality in developing countries, and it is a known leading global infectious disease. Due to the effect of MDR, XDR and TDR tuberculosis, the standard six and nine months treatment have become less active, time-consuming and expensive. This effect has led to efforts by many scientists at developing new anti-tuberculosis drugs, which would combat both the MDR and XDR tuberculosis and also minimize the treatment period, and also improve patient compliance. Therefore, it is essential to develop new antituberculosis drugs which can inhibit both actively multiplying bacilli and a non-growing persistent population of MTB to prevent reactivation of the infection.

UDP-N-acetylenolpyruvoylglucosamine reductase (MurB) is involved in the catalysis of the final steps of the UDP-N-acetylmuramic acid (UDPMurNAc) formation. This reaction is an NADPH-dependent reduction of enolpyruvyl-UDP-N-acetylglucosamine (EP-UDP-NAc), releasing UDP- $N$-acetylmuramic acid (UDP-MurNAc) as a product, to which three amino acids will subsequently be added sequentially by other enzymes in the pathway (Benson et al., 1993; Moraes et al., 2015). MurB is a flavoprotein and belongs to the superfamily category of FAD-binding protein with a feature of Flavin Adenine Dinucleotide (FAD) binding fold (Murzin, 1996). It also has FAD as a cofactor which is believed to transfer a proton from NADPH and water to FAD and later from $\mathrm{FADH}_{2}$ to EP-UDPGlcNAc. Since MurB possessed by all the classes of bacteria (gram-positive and gram-negative bacteria), compounds require for it inhibition must have broad-spectrum activity. Also, the enzyme is unique to bacteria, with no known homologous in human (Benson et al., 1993; Moraes et al., 2015). The catalytic activity of MurB divided into two half-reactions, with an enzyme-bound FAD serving as a redox intermediate. The first half begins with the binding of NADPH to the MurB, which is accompanied by hydride transfer of the 4-pro-S hydrogen of NADPH to N5 of the MurB-bound flavin, which leads to the reduction of a FAD to FADH2, with the liberation of NADP ${ }^{+}$. This process followed by the binding of EP-UDPGlcNAc. The second half-reaction is the formation of UDPMurNAc from EP-UDPGlcNAc via reduction process, 
where there is a transfer of the hydride from reduced flavin $(\text { Enz-FADH })_{2}$ to $\mathrm{C}-3$ of the enolpyruvyl moiety of the EP-UDP-GlcNAc. It leads to the formation of carbanion equivalent at $\mathrm{C}-2$ and helps to stabilize the $\alpha$-carboxylate at $\mathrm{C}-1$ as an enol intermediate. UDPMurNAc formed after the transferred of the solvent-equilibrated proton to C- 2 . The reaction catalyzes by MurB has both weak and robust substrate inhibition by NADPH and EP-UDPGlcNAc respectively, which proceed via the ping-pong mechanism (Moraes et al., 2015). The first molecule found to inhibit MurB was tri-substituted thiazolidinones which developed via parallel synthesis approach, to mimic the diphosphate moiety of the EP-UDPGlcNAc (Andres et al., 2000; Moraes et al., 2015). Many analogs of imidazoline were synthesized and exhibit the inhibitory activity against MurB as well as good antimicrobial activity against S. aureus (Bronson et al., 2003; Moraes et al., 2015). More than 195 compounds (4-alkyl and 4,4-dialkyl 1,2-bis(4-chlorophenyl)pyrazolidine-3,5-dione derivatives) were proposed to be potent inhibitors of MurB, and the majority of them showed good activity in vitro with low MIC values against gram-positive bacteria (Kutterer et al., 2005). Yang et al. (2006), identified set of 3,5-dioxopyrazolidines as novel inhibitors of MurB from their study. The 3,5-dioxopyrazolidines can bind to the active site of MurB adjacent to the FAD cofactor. These compounds are novel inhibitors not only to grampositive bacteria but also to the antibiotic-resistant strain. To date, no compounds have been reported to have inhibitory activity against MurB from MTB. Therefore, the objective of this study was to determined novel inhibitors of MurB from MTB through docking and MD simulation analyses.

\section{Materials and Methods}

\section{Preparation of Crystal Structure of MurB}

Crystal Structure of MurB from MTB (PDB Code: 5JZX and resolution $2.2 \AA$ ) bound to FAD and $\mathrm{K}^{+}$was obtained from Protein Data Bank (PDB). The structure of the protein was prepared to ensure high-qualit and reliable structure. The bound cofactors removed. The structures of protein cleaned, and missing atoms or residues were check. Missing hydrogens added, missing loops were identified and fixed, and alternate conformation was check and removed. Side chains identified and attached, inappropriate chirality ascertained, and disulfide bond and steric clashes identified and corrected. Water molecules and all nonprotein residues removed through energy minimization and protein optimization using programs implemented in Chimera (Pettersen et al., 2004) and SwissPDViewer (Johansson 
et al., 2012). The residues bound to FAD was identified by submitting the 5JZX into the Ligand Contact Tool (LCT). This program identified residues of MurB interacted with the FAD.

\section{Virtual Screeining}

Virtual screening was used to find the ligands that interact with the MurB to produce the desired therapeutic effect such as antibacterial activity. In this study, compounds from zinc database commercially available in the public domain used for virtual screening with PyRx 0.8 tool. The whole ligands were prepared using PyRx before molecular docking to obtain different binding conformation and minimum energy state. Two thousand one hundred and fifty-seven (2157) compounds with best binding conformations, and lower binding energies obtained from zinc database. These compounds further screened for drug-likeness, pharmacokinetic and physicochemical properties using AdmetSAR tool (Cheng et al., 2012), DataWarrior tool and ADME/TOX program (Lipinski et al., 2001; Veber et al., 2002) to obtained compounds with desirable properties. The physicochemical properties include molecular weight, lipophilicity, hydrogen bond donor (HBD) and hydrogen bond acceptor (HBA). While the pharmacokinetic properties analyzed in this study include Blood-Brain Barrier (BBB) penetration, Human Intestinal Absorption (HIA), Cytochrome P450 (CYP450 2D6) Inhibitor, Aqueous Solubility and Plasma Protein Binding (PBP), Mutagenicity, Tumorigenicity, Irritation, and Reproduction.

\section{Molecular Docking Analysis}

The compounds that possessed desirable properties after virtual screening were used for molecular docking analysis using Audodock4.22 (Morris et al., 1998). Molecular docking of ligands to the protein was carried out to determine the binding orientation of the proteinligand complex. A FAD which serves as a cofactor was also docked to the MurB to compare it binding energy with the selected ligands. A Lamarckian genetic algorithm was used to calculate the free energy and the RMSD were an analysis. Polar hydrogens with given Kollman charges applied for the protonation of MurB. The PDB was used to generate PDBQT which contained information of atom types, partial charges and the torsional degree of freedom. The MurB was kept in a fixed position while the ligands side chains and torsional bonds were allowed to move freely. The grid map was set at $60 \times 60 \times 60 \AA$ and with $0.375 \AA$ spacing (LaMotta et al., 2007). A total of 10 runs performed with a population size of 150, a maximum generation of 27000 and a maximum evaluation of 2, 500,000. Lastly, the binding 
energy calculated and the RMSD was analyzed. Visualization of the protein-ligand complex was performed using Pymol (1.7.4.5 Edu) Molecular Graphics System, Version 1.8 Schrödinger, LLC (DeLano, 2002) and Ligplot+ v.1.4.5 tool (Laskowski and Swindells, 2011; Wallace et al., 1996).

\section{Molecular Dynamic Simulation}

The MD simulation of the MurB complexed with the ligands was carried out using an AMBERTOOLS10 package of Molecular Dynamic(Case et al., 2008). The explicit hydrogen was added to the complex through protonate 3D. The Antechamber used to combine all missing parameters for the ligands. The topology and coordinate file of the protein-ligand complex were built using tleap. The tleap were used to assigned ff12SB and GAFF parameter for the ligand and the protein respectively. The complete system inserted into a buffer solution of $10 \AA$ of TIP3P water contained in the octahedral box neutralized by sodium ions. The system was minimized to remove by subjecting to maximum minimization cycle of 10000 steps. These include 5000 steps of minimization of conjugate gradient and 5000 steps of steepest descent, with a restraint run at $544 \mathrm{kcal} / \mathrm{mol} / \AA$ on the complexes. Then the restrained was removed, and the system minimized for 2500 steps of steepest descent with additional 2500 steps of conjugate gradients. The system heated at temperature with the initial temperature of $0.0 \mathrm{k}$ and a final temperature of $300 \mathrm{k}$ for $100 \mathrm{ps}(100,000$ steps $)$ using Langevin dynamics temperature regulation. For the first 90000 steps, the temperature increased from $0 \mathrm{~K}$ to $300 \mathrm{~K}$ and from 90001 to 100000 ; the temperature remains at $300 \mathrm{~K}$. Langevin thermostat collision frequency set at 1ps with no pressure control. The production of MD simulation was performed at the constant temperature of $300 \mathrm{~K}$ and constant pressure $1 \mathrm{~atm}$ with the time step of $2 \mathrm{fs}$, using Berendsen barostat for constant pressure simulation. A 50ns long MD simulation of the protein-ligand complex was produced with enable SHAKE to constrain all bonds involving hydrogen. The stability of the protein-ligand complex system was analyzed based on the root mean square deviation (RMSD), while the motion of specific amino acids around their mean position was determined based on the root mean square fluctuation (RMSF), to assess the flexibility of the dynamic nature of the residues during amino acid substitution. The compactness of the protein-ligand complex was checked based on the radius of gyration, to determine the degree of how folded or unfold a protein-ligand was. If the radius of gyration maintained a relatively consistent value, in the course of the MD simulation, it would regard as stably folded; otherwise, it is not (Ghasemi et al., 2016). 
All the analyses of the MD simulation were performed using ptraj in the AMBERTOOLS10 package.

\section{Results and Discussions}

\section{Virtual Screening and Molecular Docking Analysis of MurB}

MurB in MTB contained 369 amino acid residues, with a combination of $\alpha+\beta$ secondary element and it had three domains. Domain I contained residues within the range of 21-81 and 364-369amino acids; it had both $\mathrm{N}$ - and C-terminals residues, although most of the amino acids were in N-terminal portion. Similarly, domain II consisted of 90-244 residues while domain III had 251-361 amino acids. The amino acids of domains I and II were involved in FAD binding (Flavin), while domain III was involved in substrate binding. Three essential residues (Arg176, Glu361, and Ser257) in the enzyme-substrate complex and a monovalent cation were involved in the catalytic activity (Bouhss et al., 1999). Arg176 and Glu361 are located near oxygen of the enolpyruvylcarboxylate and are believed to stabilize the enol intermediate via protonation, while Ser257 involved in the transfer of a proton to an enol intermediate during the second reduction step. However, a total of eleven highly conserved residues in MurB, interact with both EP-UDP-GlcNAc and FAD, although, seven residues (Asn71, Tyr175, Arg176, Arg238, Ser257, His324, and Glu361) play an essential role in the activity (Benson et al., 1996). Therefore, inhibition of these seven amino acid residues would block the catalytic function of the MurB.

Virtual screening plays an essential role in modern drug design and discovery via screening a vast compound library for biological activity. Virtual screening can provide a molecule which is capable of binding to macromolecules such as protein and DNA with less free binding energy. Two thousand one hundred and fifty-seven (2157) compounds capable of binding to MurB with less binding energies were obtained through virtual screening against Zinc database. These compounds further filtered for physicochemical properties, drug-like properties (Table 1) and pharmacokinetic properties (Table 3). To remove compounds with undesirable properties. The compounds which possessed the desirable physicochemical propertie, pharmacokinetic, and drug-likeness properties were further used for molecular docking studies to determine their free binding energies and inhibition constant (Ki). Inhibition constant $(\mathrm{Ki})$ is the required concentration of ligand that is capable of inhibiting the protein. Therefore, a small concentration of ligand required for effective inhibition. Also, a FAD which served as a cofactor was used in the molecular docking studies to ascertain its free binding energy. Seven compounds (7) with minimum binding energies ranged between 
-11.80 and $-10.39 \mathrm{kcal} / \mathrm{mol}$ were selected, lower than the free binding energy of FAD $(-10.06 \mathrm{kcal} / \mathrm{mol})$ (Figure 1). Based on the results of the docking analyses, ZINC19837204 had the minimum binding energy of $-11.80 \mathrm{kcal} / \mathrm{mol}$ and inhibition constant $(\mathrm{Ki})$ of $2.26 \mathrm{nM}$, formed two hydrogen bonds with methyl group of Ala22 (distance $=3.29 \AA$ ) and carboxylic group of Asp246 (distance =3.17 ) (Table 2). It also undergoes hydrophobic interactions with a basic side chain of His324, which is one of the critical residues playing a pivotal role in the substrate binding site. It also interacted with many other amino acids of domains I and II, which were involved in FAD binding site (Figure 2a). Since ZINC19837204 had free binding energy, lower than the FAD, it would competitively bind to site with higher binding affinity. The ligand might also block the substrate binding site, which would inhibit the normal function of the MurB. Similarly, ZINC11839554 had the minimum binding energy of $-11.47 \mathrm{kcal} / \mathrm{mol}$ and inhibition constant $(\mathrm{Ki})$ of $3.89 \mathrm{nM}$, interacted with MurB by forming four hydrogen bonds with the carboxylic group of Asp246 (distance=2.62 $\AA$ ), the hydroxyl group of Thr248 (distance=3.27 $\AA$ ) and the non-polar side chain of Trp253 (distance=2.92 $\AA$, 3.08Å) (Table 2). Also, it undergoes hydrophobic interactions with the carboxylic group of Glu361 and polar amide of Asn71, which were the critical residues involved in the substrate binding sites of MurB (Figure 2b). ZINC14976552 had a binding affinity of $-10.77 \mathrm{kcal} / \mathrm{mol}$ and inhibition constant (Ki) of $12.81 \mathrm{nM}$, interacted and formed two hydrogen bonds with the carboxylic group of Asp246 (distance = 3.13Å) and the hydroxyl group of Thr248 (distance = 2.78Å). It also reacted in a hydrophobic way with a basic side chain of His324 (Figure 2c). ZINC18122756 possessed the binding affinity of $-10.75 \mathrm{kcal}$ and inhibition constant (Ki) of 13.20nM, interacted and formed two hydrogen bonds with the amino group of Arg238 (distance $=3.20 \AA$ ) and the hydroxyl group of Thr248 (distance $=2.91 \AA$ ). It also presented hydrophobic interaction with His324 and many other residues which were involved in the FAD-binding site (Figure 2d). Similarly, ZINC12242812 had the free binding energy of $-10.39 \mathrm{kcal} / \mathrm{mol}$ and inhibition constant $(\mathrm{Ki})$ of $22.77 \mathrm{nM}$. It interacted and formed four hydrogen bonds with the side chain hydrogen of Gly140 (distance $=3.07 \AA$ ), the carboxylic group of Asp246 (distance $=2.71 \AA$ ), the hydroxyl group of Thr248 (distance $=3.23 \AA$ ) and a carboxylic group of Glu361 (distance $=2.83 \AA \AA$ ) (Table 2). It exhibited hydrophobic interaction with a polar amide of Asn71 and amino group Arg176. Thus, ZINC12242812 is the only ligand that interacted with the three (Glu361, Asn71, and Arg176) essential residues involved in the substrate binding sites; therefore it had substantial activity against the normal function of the MurB. However, all the seven ligands (ZINC19837204, ZINC11839554, ZINC14976552, ZINC18122756, ZINC14995379, ZINC14982226, and ZINC12242812) 
interacted with both the residues involved in the substrate binding sites and FAD binding sites. Since, the ligands had free binding energies, lower than the binding energy of the FAD, they would competitively bind to the site of FAD and the substrate which would inhibit the enzyme catalytic activity.

\section{Docking Score (kcal/mol)}
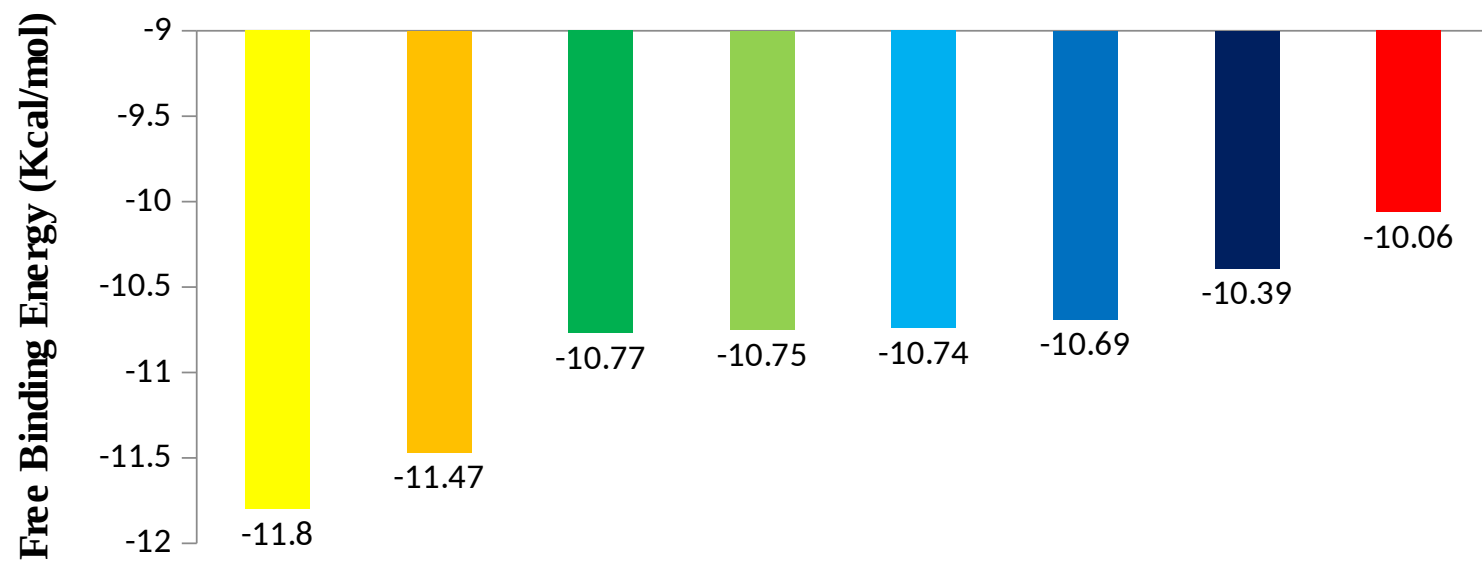

Docking Score $(\mathrm{kcal} / \mathrm{mol})$

Figure 1: Free binding energies of selected ligands interacted with MurB

Table 1: Molecular Properties and Drug-likeness of the selected ligands with MurB

\begin{tabular}{|c|l|c|c|c|c|c|}
\hline S/No. & \multicolumn{1}{|c|}{ Zinc Code } & $\begin{array}{c}\text { Molecular } \\
\text { Weight }\end{array}$ & cLogP & $\begin{array}{c}\text { H-bond } \\
\text { Acceptors }\end{array}$ & $\begin{array}{c}\text { H-bond } \\
\text { Donors }\end{array}$ & $\begin{array}{c}\text { Drug- } \\
\text { likeness }\end{array}$ \\
\hline 1. & $\begin{array}{l}\text { ZINC1983720 } \\
4\end{array}$ & 482.650 & 2.1553 & 6 & 2 & 4.414 \\
\hline 2. & ZINC11839554 & 484.618 & 2.9198 & 6 & 1 & 1.9694 \\
\hline 3. & $\begin{array}{l}\text { ZINC1497655 } \\
2\end{array}$ & 497.621 & 3.0635 & 7 & 1 & 7.4954 \\
\hline 4. & $\begin{array}{l}\text { ZINC1812275 } \\
6\end{array}$ & 488.614 & 4.3155 & 8 & 1 & 11.116 \\
\hline 5. & $\begin{array}{l}\text { ZINC1499537 } \\
9\end{array}$ & 491.654 & 1.2953 & 7 & 1 & 3.691 \\
\hline 6. & $\begin{array}{l}\text { ZINC1498222 } \\
6\end{array}$ & 495.645 & 3.0547 & 6 & 2 & 4.451 \\
\hline 7. & $\begin{array}{l}\text { ZINC1224281 } \\
2\end{array}$ & 496.678 & -0.573 & 8 & 3 & 3.4883 \\
\hline
\end{tabular}


Table 2: Free binding energies and residues of MurB involved in hydrogen bonds with the selected ligands

\begin{tabular}{|c|c|c|c|c|c|}
\hline S/No. & Zinc Code & $\begin{array}{l}\text { Docking Score } \\
\text { (kcal/mol) }\end{array}$ & $\begin{array}{l}\text { Inhibition } \\
\text { Constant Ki } \\
\text { (nM) }\end{array}$ & $\begin{array}{l}\text { Residues involved in } \\
\text { Hydrogen bonding }\end{array}$ & $\begin{array}{c}\text { Distance } \\
(\AA)\end{array}$ \\
\hline 1 & ZINC19837204 & -11.80 & 2.26 & $\begin{array}{c}\text { Ala22 } \\
\text { Asp246 }\end{array}$ & $\begin{array}{l}3.29 \\
3.17\end{array}$ \\
\hline 2. & ZINC11839554 & -11.47 & 3.89 & $\begin{array}{l}\text { Asp246 } \\
\text { Thr248 } \\
\text { Trp253 } \\
\text { Trp253 }\end{array}$ & $\begin{array}{l}2.62 \\
3.27 \\
2.92 \\
3.08\end{array}$ \\
\hline 3. & ZINC14976552 & -10.77 & 12.81 & $\begin{array}{l}\text { Asp246 } \\
\text { Thr248 }\end{array}$ & $\begin{array}{l}3.13 \\
2.78\end{array}$ \\
\hline 4. & ZINC18122756 & -10.75 & 13.20 & $\begin{array}{l}\text { Arg238 } \\
\text { Thr248 } \\
\end{array}$ & $\begin{array}{l}3.20 \\
2.91\end{array}$ \\
\hline 5. & ZINC14995379 & -10.74 & 13.36 & $\begin{array}{l}\text { Asp246 } \\
\text { Trp253 }\end{array}$ & $\begin{array}{l}2.72 \\
2.55\end{array}$ \\
\hline 6. & ZINC14982226 & -10.69 & 14.53 & Asp246 & 2.65 \\
\hline 7. & ZINC12242812 & -10.39 & 22.77 & $\begin{array}{l}\text { Gly140 } \\
\text { Asp246 } \\
\text { Thr248 } \\
\text { Glu361 }\end{array}$ & $\begin{array}{l}3.07 \\
2.71 \\
3.23 \\
2.83\end{array}$ \\
\hline
\end{tabular}


(a)
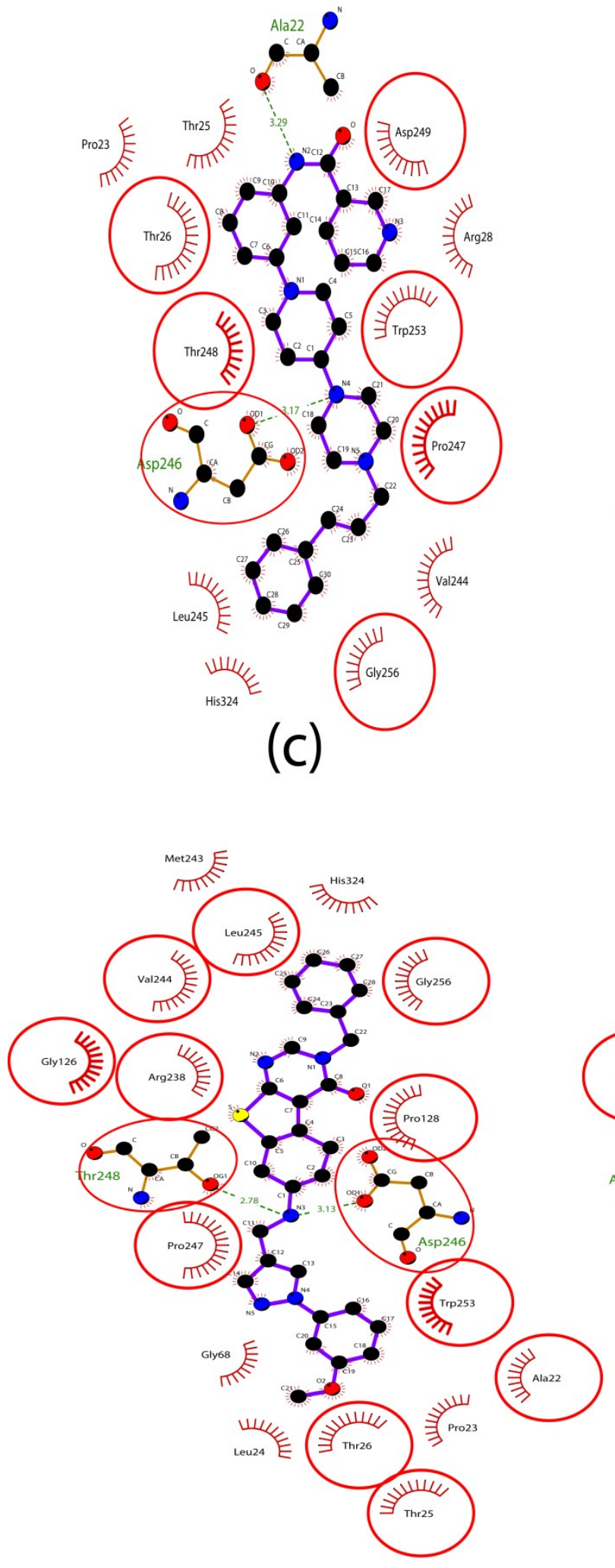

(b)
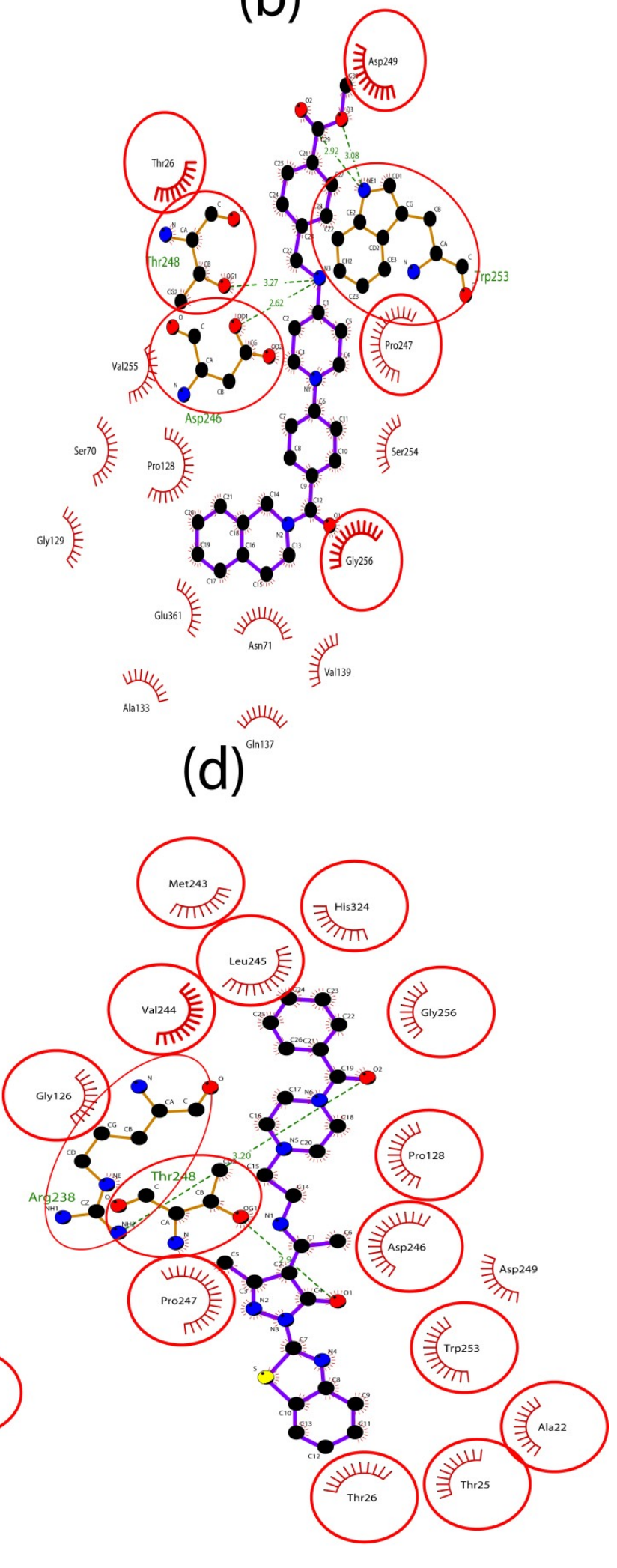
(e)
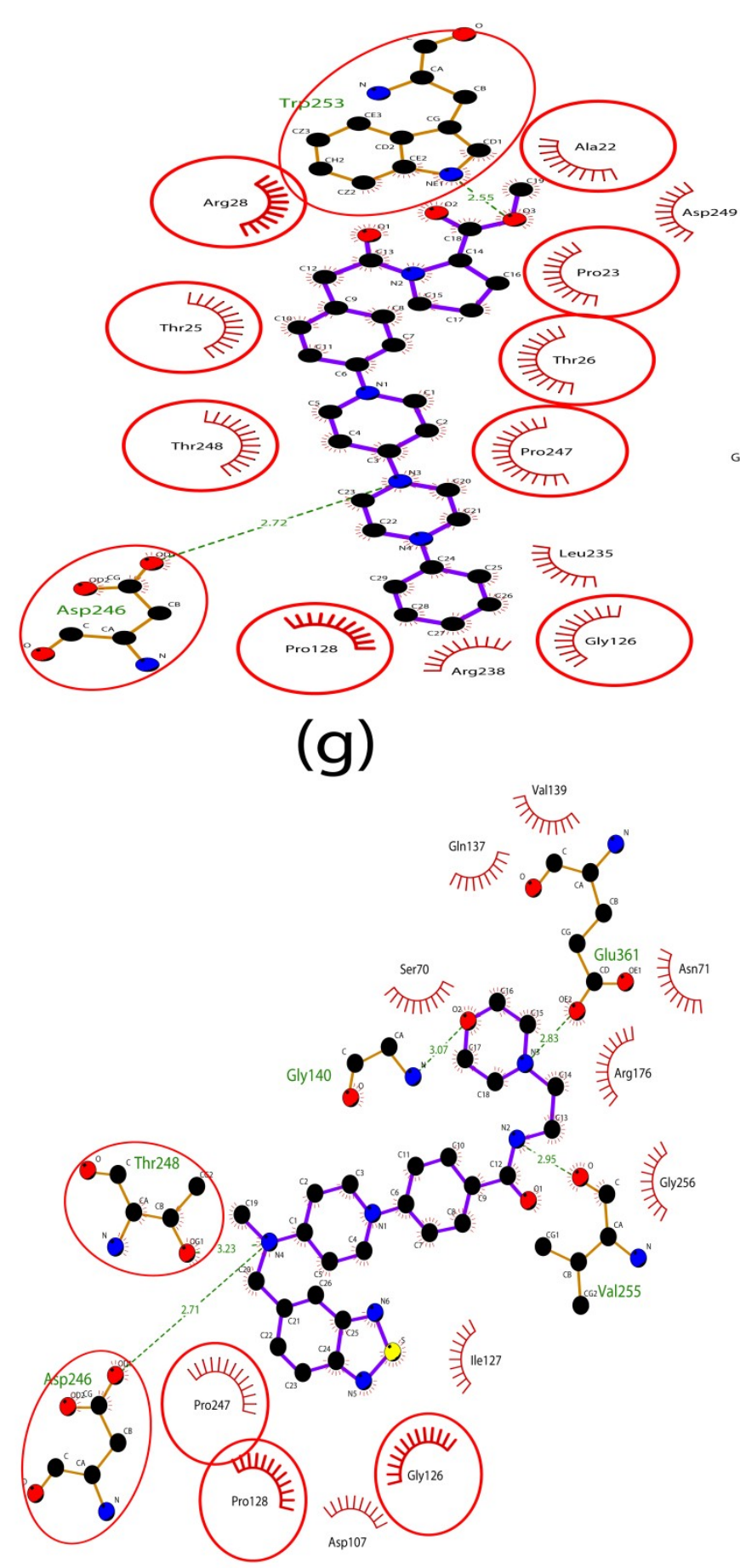

(f)

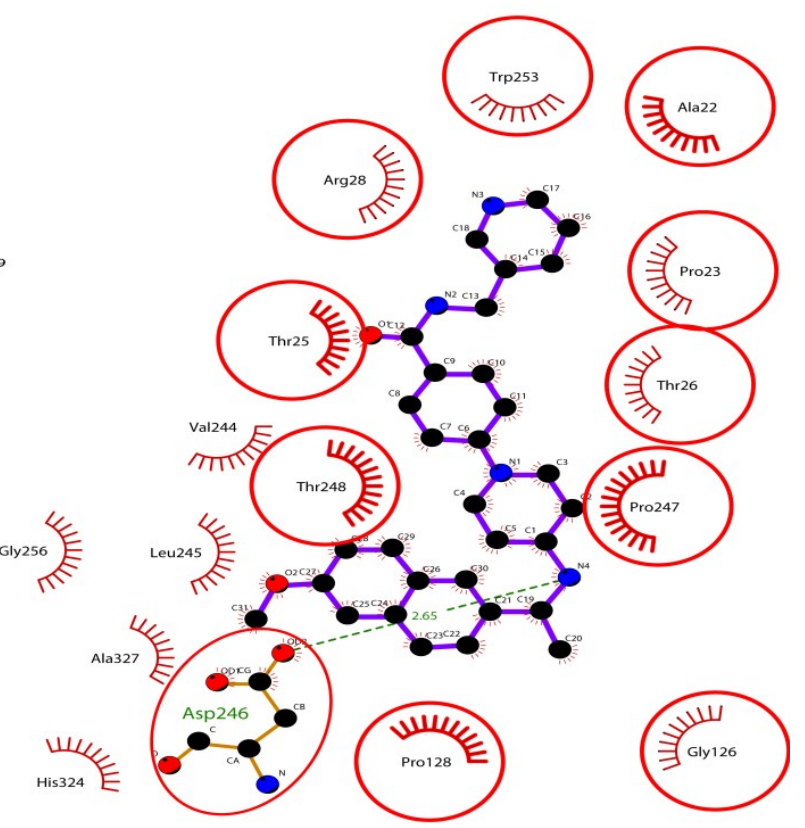

- 
Table 3: ADME and Toxicity Analyses of the Selected Ligands Interacted with MurB

\begin{tabular}{|c|l|c|c|c|c|c|c|c|c|c|c|}
\hline $\begin{array}{c}\text { S/No } \\
.\end{array}$ & $\begin{array}{c}\text { Zinc/PubChem } \\
\text { Code }\end{array}$ & HIA & BBB & $\begin{array}{c}\text { CYP450 2D6 } \\
\text { Inhibitor }\end{array}$ & PPB (\%) & $\begin{array}{c}\text { Aqueous } \\
\text { Solubility }\end{array}$ & $\begin{array}{c}\text { AMES Test } \\
\text { Carcinogens }\end{array}$ & $\begin{array}{c}\text { Mutag } \\
\text { enic }\end{array}$ & $\begin{array}{c}\text { Tumorige } \\
\text { nic }\end{array}$ & $\begin{array}{c}\text { Reprod } \\
\text { ucibility }\end{array}$ \\
\hline 1 & ZINC19837204 & HIA+ & BBB+ & Non-inhibitor & 65.0492 & -3.965 & Non AMES toxic & Non-carcinogens & none & none & none \\
\hline 2 & ZINC11839554 & HIA+ & BBB+ & Non-inhibitor & 75.2633 & -4.98 & Non AMES toxic & Non-carcinogens & none & none & none \\
\hline 3 & ZINC14976552 & HIA+ & BBB+ & Non-inhibitor & 82.6130 & -5.086 & Non AMES toxic & Non-carcinogens & none & none & none \\
\hline 4 & ZINC18122756 & HIA+ & BBB+ & Non-inhibitor & 57.9290 & -3.543 & Non AMES toxic & Non-carcinogens & none & none & none \\
\hline 5 & ZINC14995379 & HIA+ & BBB+ & Non-inhibitor & 42.3557 & -3.528 & Non AMES toxic & Non-carcinogens & none & none & none \\
\hline 6 & ZINC14982226 & HIA+ & BBB+ & Non-inhibitor & 85.4859 & -5.752 & Non AMES toxic & Non-carcinogens & none & none & none \\
\hline 7 & ZINC12242812 & HIA+ & BBB+ & Non-inhibitor & 33.7113 & 0.711 & Non AMES toxic & Non-carcinogens & none & none & none \\
\hline
\end{tabular}

$\mathrm{BBB}+=$ Blood-Brain Barrier positive, BBB- = Blood-Brain Barrier negative, HIA + = Human Intestinal Absorption positive and HIA- = Human Intestinal Absorption negative, PPB = Plasma Protein Binding, Aqueous Solubility $=$ Insoluble $<-10<$ Poorly soluble $<-6<$ Moderately soluble $<-4<$ Soluble $<$ $-2<$ Very soluble $<0<$ Highly soluble 


\subsubsection{Molecular Dynamic Simulation Studies of MurB-Ligand Complexes}

Based on the result of molecular docking analysis, seven compounds (ZINC19837204, ZINC11839554, ZINC14976552, ZINC18122756, ZINC14995379, ZINC14982226 and ZINC12242812) with good binding affinities, lower than the binding energy of the FAD (cofactor) obtained. Four compounds with best binding energies (ZINC19837204 = $-11.80 \mathrm{kcal} / \mathrm{mol}$, ZINC11839554 $=-11.47 \mathrm{kcal} / \mathrm{mol}$, ZINC14976552 $=-10.77 \mathrm{kcal} / \mathrm{mol}$ ) and ability to interact with the residues (ZINC12242812 $=-10.39 \mathrm{kcal} / \mathrm{mol}$ ) in the substrate binding site further selected for the MD simulation analysis. The MD simulation was carried out to understand the stability and orientation of the selected ligands within the binding cavity of MurB, which would allow the prediction of conformational changes of both the MurB and the ligands during the MD simulation. Also, MD simulation of Apo-enzyme bound to cofactor was carried out, to compare their stability with the selected ligands. The stability of the selected complexes (MurB-ZINC19837204, MurB-ZINC11839554, MurB-ZINC14976552, and MurB-ZINC12242812) was determined by carefully examining the root mean square deviation (RMSD) during the 50ns MD simulation (Figure 3). To determine the deviation of the selected ligands concerning the binding free energy of their complexes, and movement of each residue within the protein-ligand complex, root mean square fluctuation (RMSF) was analyzed after the 50ns MD simulation. Compactness of the protein-ligand complex was checked by determining how folded or unfolded the complex was, via a radius of gyration (Ghasemi et al., 2016). The RMSD of MurB-ZINC19837204 and MurB-ZINC14976552 complexes equilibrated at 5ns and 10ns respectively and steadily moved throughout the 50ns. The complexes (MurB-ZINC19837204 and MurB-ZINC14976552) stabilized with the average mean values of $5.5242 \pm 0.016 \AA$ and $5.1862 \pm 0.018 \AA$ respectively, although higher than the mean value of FAD, which achieved stability at the average value of $3.7309 \pm 0.017 \AA$. However, both complexes achieved stability under the condition of giving MD simulation. Similarly, MurB-ZINC11839554 and MurB-ZINC12242812 complexes equilibrated at 2ns and moved throughout the 50ns, with the mean values of $4.1780 \pm 0.0101 \AA$ and $3.7361 \pm 0.0126 \AA$ respectively. Both the complexes achieved stability with average mean values closed to the mean value of FAD (cofactor), although, the MurB-ZINC12242812 complex had almost the same mean value with the cofactor (Figure 3). Therefore, all the four ligands achieved high stability and low flexibility within the binding pocket of the MurB. These high stabilities and low flexibility occurred probably as a result of interactions of the ligands with the flexible loop regions of 
MurB, which increased the stability and reduced the flexibility of the complexes (Figure 3). The fluctuation of the individual residues and the residues within the binding site of the MurB was analyzed based on RMSF. In the MurB-ZINC19837204 complex, domain I and Flavin binding domain had less flexibility, when compared with domains II and III. The range of residues fluctuations in domain I was between $7-9 \AA$, while flavin binding domain had

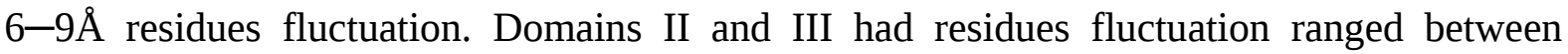

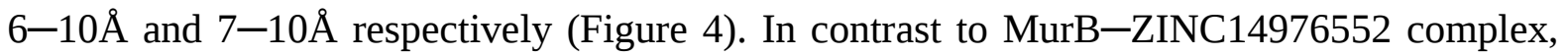
residues fluctuation in domain I ranged between 3-5A.. However, domain II and III had a similar level of fluctuations ranged between $4-10 \AA$ and $3-10 \AA$ respectively, although Arg169 and Ala277 in domain II fluctuated with the high value up to $10 \AA$. Similarly, MurB-ZINC11839554 and MurB-ZINC12242812 complexes had similar levels of

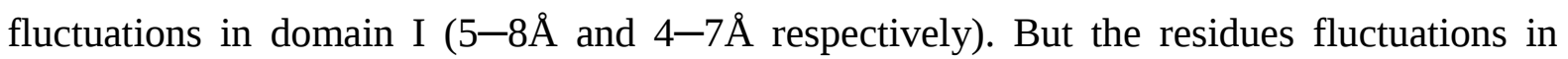
domains II and III of the MurB-ZINC11839554 complex were less than MurB-ZINC12242812 complex (Figure 4). However, all the four ligands had less flexibility within the residues involved in the substrate binding sites (Asn71, Tyr175, Arg176, Arg238, Ser257, His324, and Glu361) of the MurB as shown in Figure 6. The residues interacted with ZINC19837204 had residues fluctuation of $7 \AA$ within the substrate binding pocket, while the residues interacted with the remaining ligands had less residues fluctuations ranged between 3-5 $\AA$ (Figure 6). However, the main secondary structural element of all the complexes (MurB-ZINC19837204, MurB-ZINC11839554, MurB-ZINC14976552, and MurB-ZINC12242812) remained close to their initial structures. It had shown in Figure 7, where the complexes (after MD simulation) were superimposed on their respective initial structures (before MD simulation) and had RMSF values ranged between 2-5Å (Figure 7). It suggested that all the ligands bound to the site closed to the initial binding sites during the 50ns MD simulation. A radius of gyration of all the complexes was determined, to analyze whether the protein-ligand complexes were stably folded or unfolded during 50ns MD simulation. If the radius of gyration moved in a steady state with relatively constant values, it was regarded as stably folded and vice versa. In Figure 5, is shown that all the four complexes; MurB-ZINC19837204, MurB-ZINC11839554, MurB-ZINC14976552 and MurB-ZINC12242812 moved with relatively constant values throughout the 50ns MD simulation, with the mean values of $22.5422 \AA, 21.9481 \AA, 22.6606 \AA$ and $21.7673 \AA$ respectively. These values closed to the average value of the FAD (22.2448 $\AA$ ). Therefore, all the complexes formed relatively stable folded polypeptide structure during the 50ns MD simulation (Figure 5). 


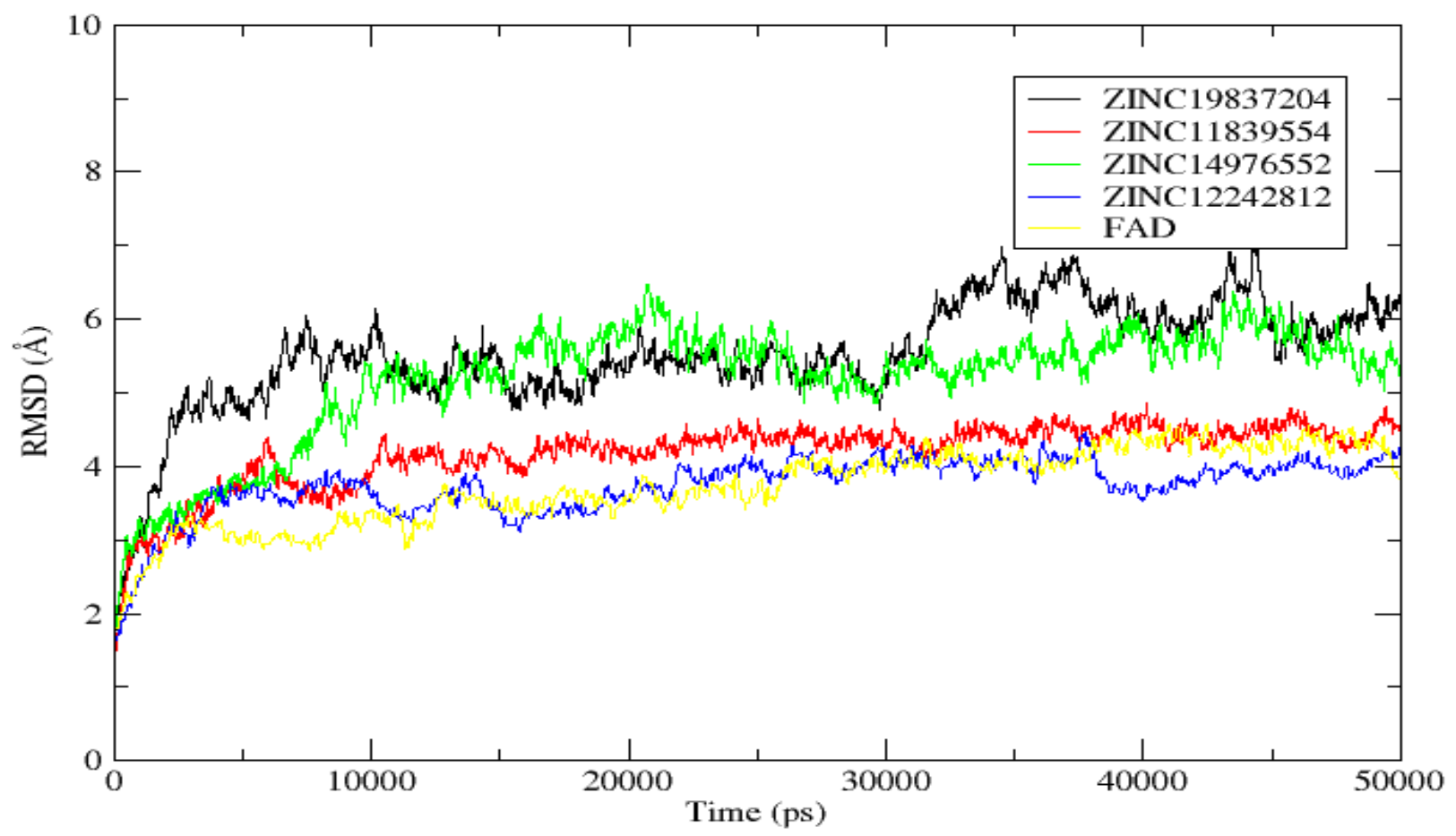

Figure 3: The MD simulation (RMSD analysis) of MurB-ZINC19837204, MurB-ZINC11839554, MurB-ZINC14976552, MurB-ZINC12242812 and MurB-FAD complexes for $50 \mathrm{~ns}$

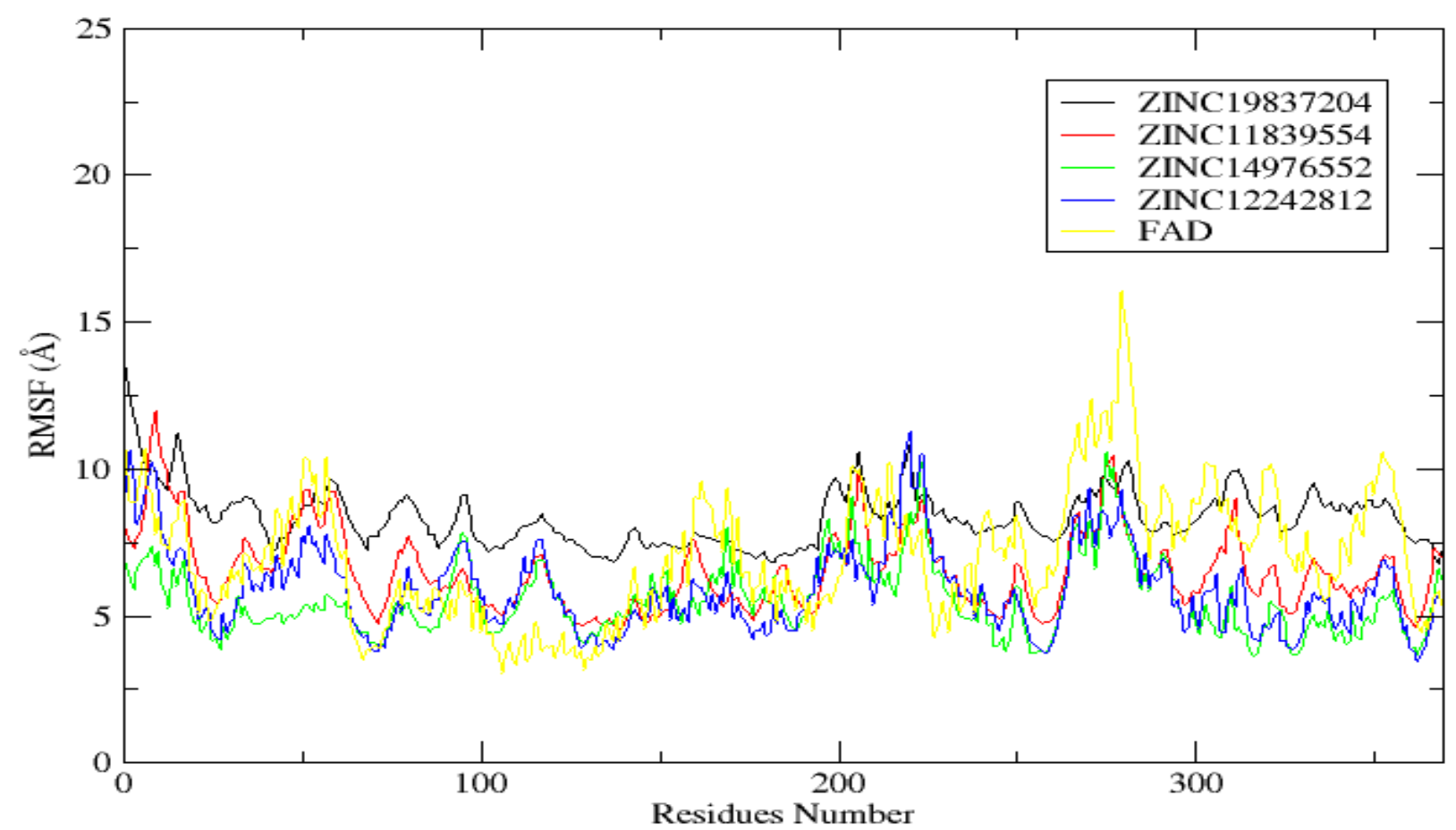

Figure 4: The MD simulation (RMSF analysis) of MurB-ZINC19837204, MurB-ZINC11839554, MurB-ZINC14976552, MurB-ZINC12242812 and MurB-FAD complexes for $50 \mathrm{~ns}$ 


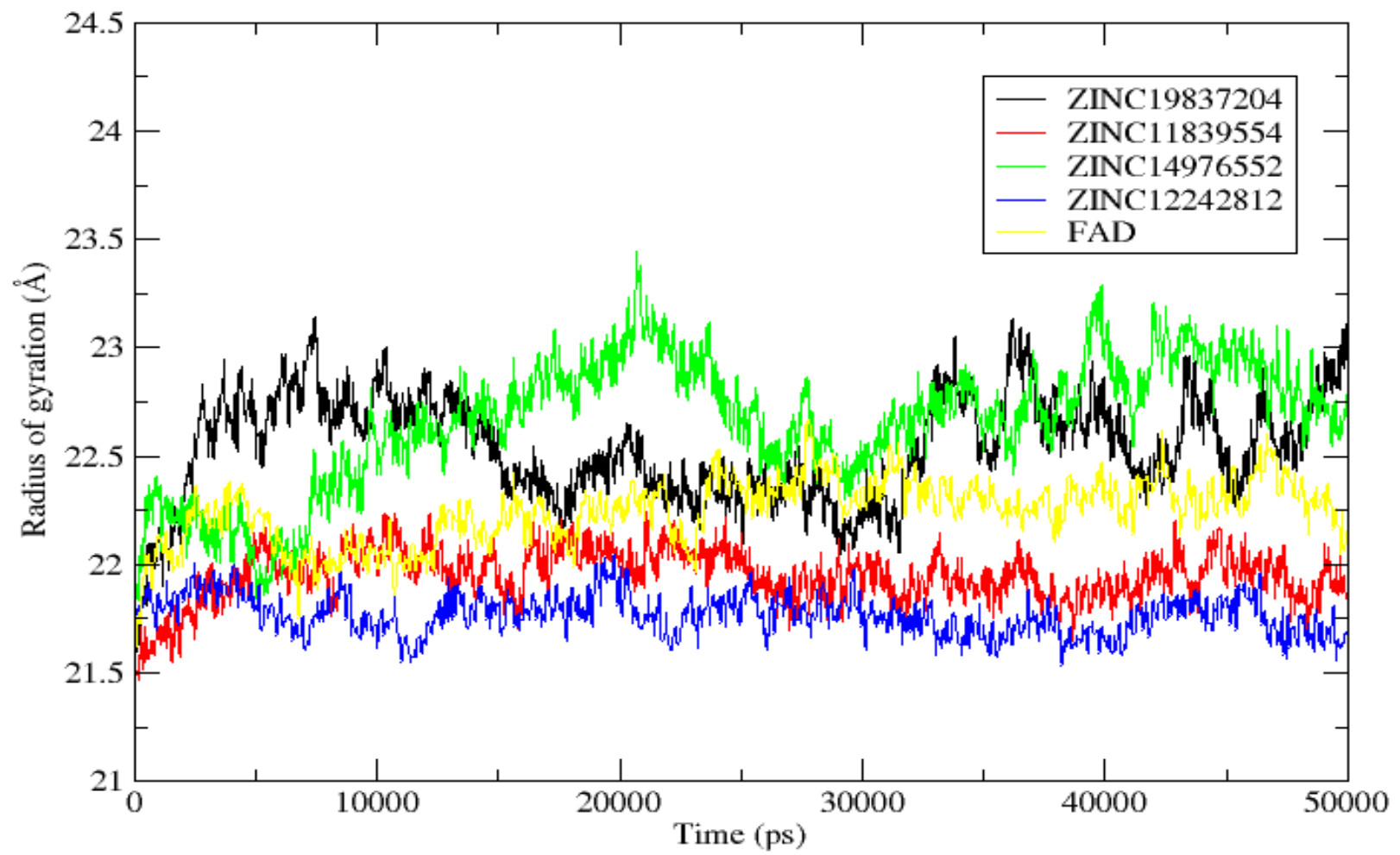

Figure 5: The MD simulation (Radius of gyration analysis) of MurB-ZINC19837204, MurB-ZINC11839554, MurB-ZINC14976552, MurB-ZINC12242812 and MurB-FAD complexes for $50 \mathrm{~ns}$

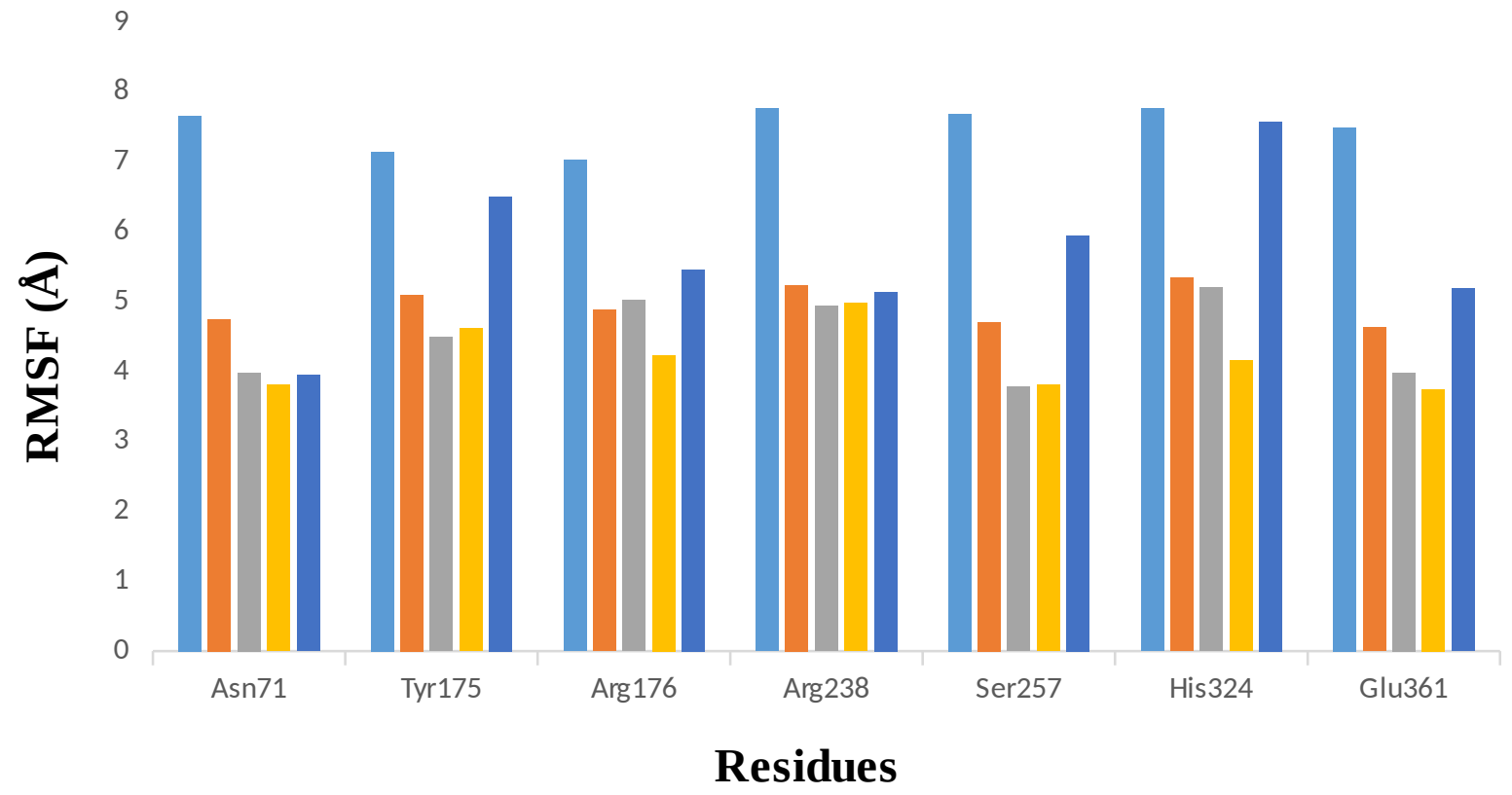

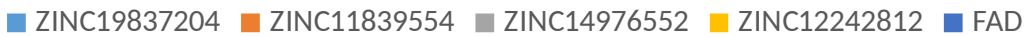

Figure 6: Residues fluctuations of MurB binding pocket after 50ns MD simulation 


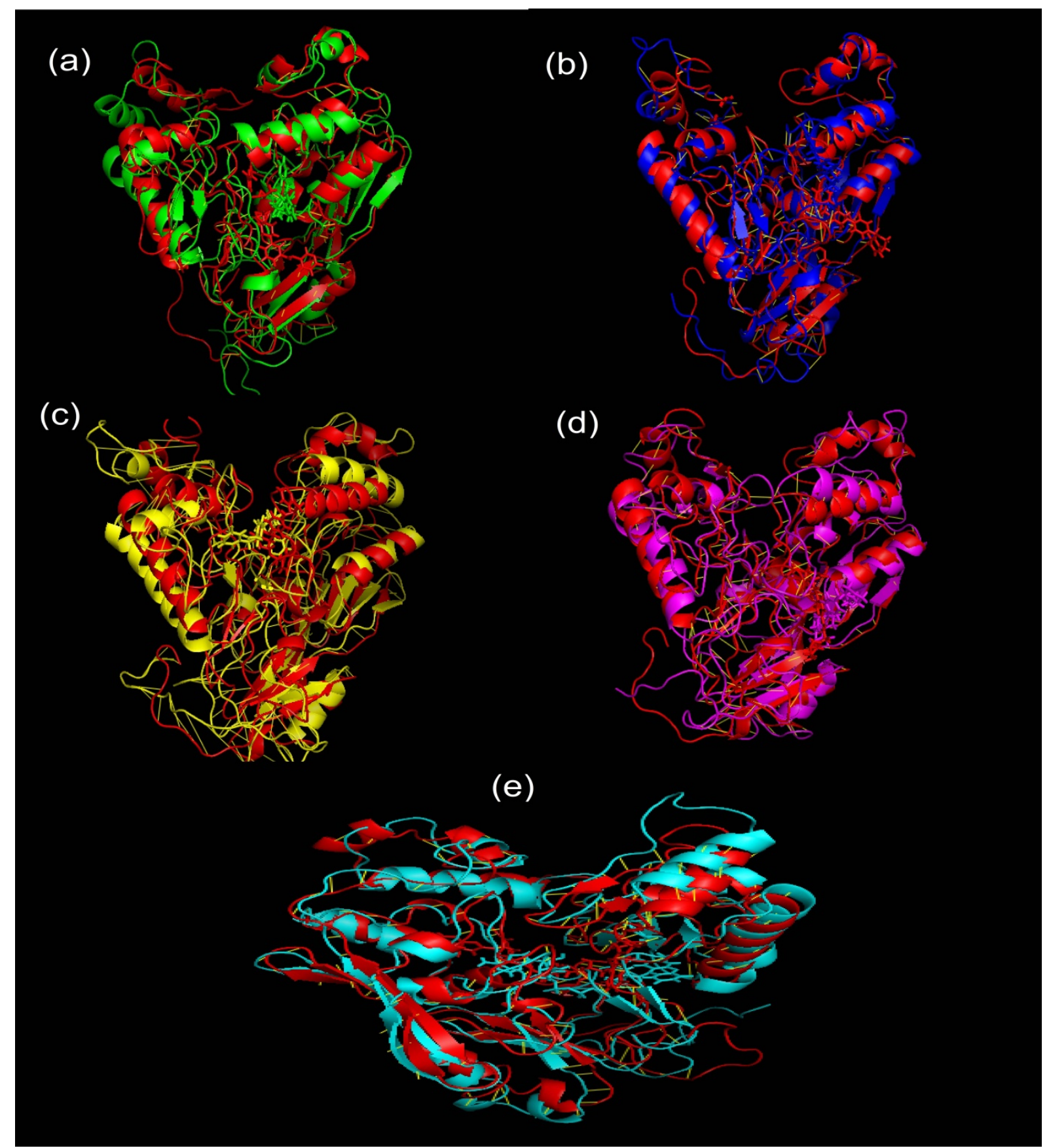

Figure 7: Superimposition of the initial complex structures (before MD simulation), and final complex structures (After MD simulation) obtained from 50ns MD simulation. (a) MurB-ZINC11839554 complex. The final structure is shown in green, and the initial

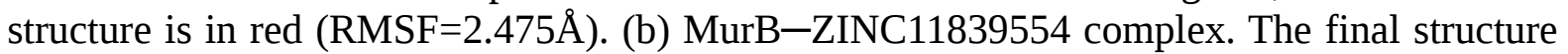
is shown in blue, and the initial structure is in red (RMSF=2.476 $\AA$ ). c) MurB-ZINC14976552 complex. The final structure is shown in yellow, and the initial structure is in red (RMSF=5.013 $\AA$ ). d) MurB-ZINC12242812 complex. The final structure is shown in purple, and the initial structure is in red (RMSF $=2.638 \AA$ ) (e) MurB-FAD complex. The final structure is shown in Cyans, and the initial structure is in red (RMSF = $2.558 \AA)$ 


\section{Free Binding Energy (MM-GBSA) Analysis}

The MM-GBSA technique is a vital method for calculating the binding energy of the protein and the ligand complex. The free binding energy of MurB and the ligands complex determine via the MM-GBSA technique present in Amber 14. The binding energy was determined using the values of the gas-phase electrostatic energy $\left(E_{\text {ele }}\right)$, van der Waals $\left(E_{v d W}\right)$, polar $\left(G_{\text {polar }}\right)$ and nonpolar $\left(G_{\text {nonpolar }}\right)$ constituent of the MurB and the ligands complex. The results of the study shown that all the ligands had the free binding energy better than the FAD $(-18.01 \pm 0.4732 \mathrm{kcal} / \mathrm{mol})$ except ZINC19837204 which has the binding energy of $-16.25 \pm 0.2416 \mathrm{kcal} / \mathrm{mol}$. This result strengthened the output of the docking analysis where the ligands had the binding energy less than the FAD. Also, polar energy supported positively to the total system energy whereas gas-phase electrostatic energy, van der Waals, and nonpolar energy component negatively underwrote to the system energy (Table 4).

Table 4: Free Binding Energy using MM-GBSA

\begin{tabular}{|c|c|c|c|c|c|}
\hline & $\Delta \mathbf{G}_{\mathbf{v d w}}$ & $\Delta \mathbf{G}_{\text {ele }}$ & $\Delta \mathbf{G}_{\text {polar }}$ & $\Delta \mathbf{G}_{\text {nonpolar }}$ & $\Delta \mathbf{G}_{\text {MM-GBSA }}$ \\
\hline ZINC1983720 & $-34.01 \pm 0.456$ & $-152.35 \pm 2.3569$ & $174.46 \pm 3.5671$ & $-4.35 \pm 0.6711$ & $\mathbf{- 1 6 . 2 5} \pm \mathbf{0 . 2 4 1}$ \\
$\mathbf{4}$ & 7 & & & & $\mathbf{6}$ \\
\hline ZINC11839554 & $-53.44 \pm 0.422$ & $-14.5339 \pm 1.0585$ & $45.34 \pm 0.8731$ & $-5.84 \pm 0.0355$ & $-\mathbf{2 8 . 4 7} \pm \mathbf{0 . 5 4 4}$ \\
& 3 & & & & $\mathbf{0}$ \\
\hline ZINC1497655 & $-42.76 \pm 0.569$ & $-16.81 \pm 0.9661$ & $44.23 \pm 0.7241$ & $-6.12 \pm 0.0781$ & $-\mathbf{2 1 . 4 5} \pm \mathbf{0 . 6 4 3}$ \\
$\mathbf{2}$ & 1 & & & & $\mathbf{1}$ \\
\hline ZINC1224281 & $-52.68 \pm 0.567$ & $-21.34 \pm 0.9612$ & $45.46 \pm 0.8761$ & $-3.04 \pm 0.0612$ & $-\mathbf{3 1 . 6 0} \pm \mathbf{0 . 7 2 5}$ \\
$\mathbf{2}$ & 8 & & & & $\mathbf{8}$ \\
\hline FAD & $-21.01 \pm 0.390$ & $-134.57 \pm 2.8560$ & $140.44 \pm 2.66$ & $-2.87 \pm 0.042$ & $\mathbf{- 1 8 . 0 1} \pm \mathbf{0 . 4 7 3}$ \\
& 6 & & & & $\mathbf{2}$ \\
\hline
\end{tabular}

\section{Conclusion}

In this study 2157 compounds obtained from two public databases, through virtual screening and use for molecular docking analysis. A total of seven compounds with suitable binding affinity and possessed all the ADME and toxicity properties. Out of this seven ligands, four ligands ((ZINC19837204, ZINC11839554, ZINC14976552, and ZINC1224281) in addition to the cofactor (FAD)), with good binding energy were selected and use for MD simulation 
analysis. The result of the MD simulation revealed that all the four ligands (ZINC19837204, ZINC11839554, ZINC14976552, and ZINC1224281) formed stable complexes in the binding site of the MurB, during the 50ns MD simulation, when compared with the cofactor (FAD). Therefore, these compounds were proposed to be potential inhibitors of MurB, after experimental validation.

\section{Conflicts of interest}

I declare that we have no conflict of interest.

\section{Acknowledgments}

The corresponding author of this paper is very much grateful to Prof. Pawan Dhar (Jawaharlal Nehru University), Prof. B. Jayaram (Coordinator of the Supercomputing Facility for Bioinformatics \& Computational Biology, IIT Delhi), Dr. Kalaiarasan P. (Jawaharlal Nehru University), and Mr. Shashank Shekhar (IIT Delhi) for their contribution and providing facilities. 


\section{References}

Andres C. J., Bronson J. J., D'Andrea S. V., Deshpande M. S., Falk P. J., Grant-Young K. A., Harte W. E., Ho H. T., Misco P. F., Robertson J. G., Stock D., Sun Y. X. and Walsh A. W. (2000). 4- Thiazolidinones: novel inhibitors of the bacterial enzyme MurB. Bioorg Med Chem Lett. 10:715e7. http://dx.doi.org/10.1016/s0960-894x(00) 00073-1.

Benson, T. E., Marquardt, J. L., Marquardt, A. C., Etzkorn, F. A., \& Walsh, C. T. (1993). Overexpression, purification, and mechanistic study of UDP-Nacetylenolpyruvylglucosamine reductase. Biochemistry, 32(8), 2024-2030.

Benson, T. E., Walsh, C. T., \& Hogle, J. M. (1996). The structure of the substrate-free form of MurB, an essential enzyme for the synthesis of bacterial cell walls. Structure, 4(1), 47-54.

Bronson, J. J., DenBleyker, K. L., Falk, P. J., Mate, R. A., Ho, H. T., Pucci, M. J., \& Snyder, L. B. (2003). Discovery of the first antibacterial small molecule inhibitors of MurB. Bioorganic \& medicinal chemistry letters, 13(5), 873-875.

Bouhss, A., Dementin, S., van Heijenoort, J., Parquet, C., \& Blanot, D. (1999). Formation of adenosine 5'-tetraphosphate from the acyl phosphate intermediate: a difference between the MurC and MurD synthetases of Escherichia coli. FEBS letters, 453(1-2), 15-19.

Case DA, Darden TA, Cheatham III TE, Simmerling CL, Wang J, Duke RE, Luo R, Crowley M, Walker RC, Zhang W, Merz KM, Wang B, Hayik S, Roitberg A, Seabra G, Kolossváry I, Wong KF, Paesani F, Vanicek J,Wu X, Brozell SR, Steinbrecher T, Gohlke H, Yang L, Tan C, Mongan J, Hornak V, Cui G, Mathews DH, Seetin MG, Sagui C, Babin V, Kollman PA(2008) AMBER 10.University of California, San Francisco

Cheng, F., Li, W., Zhou, Y., Shen, J., Wu, Z., Liu, G., ... \& Tang, Y. (2012). AdmetSAR: a comprehensive source and free tool for assessment of chemical ADMET properties.

DeLano, W. L. (2002). The PyMOL user's manual. DeLano Scientific, San Carlos, CA, 452.

Ghasemi, F., Zomorodipour, A., Karkhane, A. A., \& Khorramizadeh, M. R. (2016). In silico designing of hyper-glycosylated analogs for the human coagulation factor IX. Journal of Molecular Graphics and Modelling, 68, 39-47.

Johansson MU, Zoete V,Michielin O, Guex N (2012) Defining and searching for structural motifs using DeepView/Swiss-PdbViewer.BMC Bioinf 13(1):173. 
Jothieswari, D., \& Bhaskar Reddy, K. (2015). Molecular Docking studies of potential chemical inhibitors on multi-drug resistance genes in MTB. International Journal of Innovative Drug Discovery, 5(1), 40-45.

Kutterer, K. M., Davis, J. M., Singh, G., Yang, Y., Hu, W., Severin, A., ... \& Katz, A. H. (2005). 4-Alkyl and 4, 4'-dialkyl 1, 2-bis (4-chlorophenyl) pyrazolidine-3, 5-dione derivatives as new inhibitors of bacterial cell wall biosynthesis. Bioorganic \& medicinal chemistry letters, 15(10), 2527-2531.

La Motta, C., Sartini, S., Mugnaini, L., Simorini, F., Taliani, S., Salerno, S., ... \& Cantore, M. (2007). Pyrido [1, 2-a] pyrimidine-4-one derivatives as a novel class of selective aldose reductase inhibitors exhibiting antioxidant activity. Journal of Medicinal Chemistry, 50(20), 4917-4927.

Laskowski, R. A., \& Swindells, M. B. (2011). LigPlot+: multiple ligand-protein interaction diagrams for drug discovery.

Lipinski, C. A., Lombardo, F., Dominy, B. W., \& Feeney, P. J. (2001). Experimental and computational approaches to estimate solubility and permeability in drug discovery and development settings1. Advanced drug delivery reviews, 46(1-3), 3-26.

Moraes, G. L., Gomes, G. C., De Sousa, P. R. M., Alves, C. N., Govender, T., Kruger, H. G., ... \& Lameira, J. (2015). Structural and functional features of enzymes of MTB peptidoglycan biosynthesis as targets for drug development. Tuberculosis, 95(2), 95111.

Murzin, A. G. (1996). Structural classification of proteins: new superfamilies. Current opinion in structural biology, 6(3), 386-394.

Morris, G. M., Goodsell, D. S., Halliday, R. S., Huey, R., Hart, W. E., Belew, R. K., \& Olson, A. J. (1998). Automated docking using a Lamarckian genetic algorithm and an empirical binding free energy function. Journal of computational chemistry, 19(14), 1639-1662.

Pettersen, E. F., Goddard, T. D., Huang, C. C., Couch, G. S., Greenblatt, D. M., Meng, E. C., \& Ferrin, T. E. (2004). UCSF Chimera-a visualization system for exploratory research and analysis. Journal of computational chemistry, 25(13), 1605-1612.

Veber, D. F., Johnson, S. R., Cheng, H. Y., Smith, B. R., Ward, K. W., \& Kopple, K. D. (2002). Molecular properties that influence the oral bioavailability of drug candidates. Journal of medicinal chemistry, 45(12), 2615-2623.

Wallace, A. C., Laskowski, R. A., \& Thornton, J. M. (1996). Derivation of 3D coordinate templates for searching structural databases: Application to Ser-His-Asp catalytic triads in the serine proteinases and lipases. Protein Science, 5(6), 1001-1013.

World Health Organization (2016). Global Tuberculosis Report (http://www.who.int/tb/publications/global report/en//http://apps.who.int/iris/bitstrea m/10665/250441/1/9789241565394-eng.pdf?ua=1, access, 12 May 2017). 
Yang, Y., Severin, A., Chopra, R., Krishnamurthy, G., Singh, G., Hu, W., ... \& Shlaes, D. M. (2006). 3, 5-Dioxopyrazolidines, novel inhibitors of UDP-Nacetylenolpyruvylglucosamine reductase (MurB) with activity against Gram-positive bacteria. Antimicrobial agents and chemotherapy, 50(2), 556-564. 